\title{
About Gotô's method showing surjectivity of word maps
}

\author{
Abdelrhman Elkasapy and Andreas Thom
}

\begin{abstract}
Let $\mathbf{F}$ be the free group on two letters. For $\omega \in \mathbf{F}$ we study the associated word map $\omega: S U(n) \times S U(n) \rightarrow S U(n)$. Extending a method of Gotô, we show that for $\omega$ not in the second derived subgroup $\mathbf{F}^{(2)}$ of $\mathbf{F}$, there are infinitely many $n \in \mathbb{N}$ such that the associated word map $\omega: S U(n) \times S U(n) \rightarrow S U(n)$ is surjective.
\end{abstract}

\section{Introduction}

Let $\mathbf{F}$ be the free group on two letters and let $\omega \in \mathbf{F}$. The word map $\omega: S U(n) \times S U(n) \rightarrow$ $S U(n)$ is the natural map, which is given by evaluating $\omega$ on the pair of matrices in $S U(n)$. It has been asked by Michael Larsen at the 2008 Spring Central Section Meeting of the AMS in Bloomington whether for every non-trivial $\omega \in \mathbf{F}$ and $n \in \mathbb{N}$ high enough, the associated word map $\omega: S U(n) \times S U(n) \rightarrow S U(n)$ is surjective. The aim of this work is to provide evidence for a positive answer to this question and prove the surjectivity for some classes of word maps. For convenience we restrict our study to the case $S U(n)$, even though our methods extend to other compact Lie groups.

Questions about the size of the image of word maps for general groups $G$ (in place of $S U(n)$ ) have a long history and led to interesting connections with various fields of mathematics. The first result of general type is a theorem of Amand Borel [2] asserting that any non-trivial word map is dominant (as a map between affine complex algebraic varieties) if $G$ is a simple algebraic group; in particular its image is Zariski dense. Despite this general result, the images of word maps can be very small for compact groups. Indeed, the second author showed in [10] that for fixed $n \in \mathbb{N}$ and any neighborhood $U$ of $1_{n} \in S U(n)$, there exists $\omega \in \mathbf{F} \backslash\{e\}$ such that the image of the associated word maps is contained in $U$. This result is already non-trivial for $n=2$ and led to answers to various long-standing questions in non-commutative harmonic analysis [10].

Recently, there has been an extensive study of the size of word maps for finite simple groups, see [4-6] and the references therein. One of the high points was the proof of the Ore conjecture [7], asserting that every element in a non-abelian finite simple group is a commutator.

Let us come back to $G=S U(n)$. We will observe that Larsen's question becomes more complicated if $\omega \in \mathbf{F}$ lies deeper in the lower central series. As usual, we define the lower central series by $\mathbf{F}^{(0)}:=\mathbf{F}$ and $\mathbf{F}^{(k+1)}:=\left[\mathbf{F}^{(k)}, \mathbf{F}^{(k)}\right]$. It is easy to see that for $\omega \notin \mathbf{F}^{(1)}, \omega: S U(n) \times S U(n) \rightarrow S U(n)$ 
is surjective, see for example Lemma 2.1. Hence, the first non-trivial case is $w(a, b)=[a, b]:=$ $a b a^{-1} b^{-1}$, the commutator of the generators of $\mathbf{F}$. This case - unlike for finite simple groups was solved by Tôyama already in 1949. He proved that any element in $S U(n)$ can be written as a commutator $[u, v]$ for suitably chosen elements $u, v \in S U(n)$, see [11] for more details. In the same year Gôto put this result in a more general framework, see [3]. We will recall Gotô's proof and will take Gotô's method as the basis for the proof of our result which covers all words $\omega \notin \mathbf{F}^{(2)}$. For $n \in \mathbb{N}$, we denote by $\operatorname{lpf}(n)$ the least prime factor of $n$. Our main result is the following:

Theorem 1.1. Let $\mathbf{F}$ be the free group on two generators and $\omega \in \mathbf{F}$. If $\omega \notin \mathbf{F}^{(2)}$, then there exists an integer $k \in \mathbb{N}$, such that for all $n \in \mathbb{N}$ with $\operatorname{lpf}(n) \geq k$, the word map $\omega: S U(n) \times S U(n) \rightarrow$ $S U(n)$ is surjective.

It is not known to us if the restriction on the integer $n \in \mathbb{N}$ in the assumptions of the previous theorem is necessary. We will prove Theorem 1.1 at the end of Section 3. For particular words we can say more. We define the sequence of Engel words by

$$
e_{0}(a, b)=a, \quad e_{k}(a, b)=\left[e_{k-1}, b\right], \text { for } k \geq 1 .
$$

It is easy to see that $e_{k} \notin \mathbf{F}^{(2)}$ for all $k \in \mathbb{N}$ so that the previous theorem applies. However, in this case we can show:

TheOrem 1.2. For all $k, n \in \mathbb{N}$, the $k$-th Engel word map $e_{k}: S U(n) \times S U(n) \rightarrow S U(n)$ is surjective.

This result complements results for finite simple groups of Lie type which were obtained by Bandman-Grunewald-Garion in [1]. We will prove Theorem 1.2 in the beginning of Section 4.

The article is structured as follows. Section 2 contains some preliminaries on the combinatorics of the free group and on Lie theory. We also review some known results concerning word maps and present a streamlined form of Gotô's proof from 1949. Section 3 contains the proof of the main result. Here, we have to go into some tedious computations with commutators which we think are necessary to control the effect of a base change in the free group on the natural basis of the natural basis of the derived subgroup. In Section 4 contains a study of Engel words and ends with some questions we could not answer so far.

This article contains work which is part of the PhD-project of the first author.

\section{Preliminaries and review of known results}

2.1. The free group. Let us fix some notation. Let $\mathbf{F}$ be the free group on two generators $a$ and $b$. A word in $a, b$ takes the form $a^{n_{1}} b^{m_{1}} \cdots a^{n_{k}} b^{m_{k}}$ for $n_{i}, m_{i} \in \mathbb{Z}$. It is well known that $\mathbf{F}^{(1)}$ is the free group on the set $S:=\left\{\left[a^{n}, b^{m}\right] \mid n, m \in \mathbb{Z}, n m \neq 0\right\}$. Indeed, this is a special case of Proposition 4 in Chapter I, $\$ 1.3$ of $[\mathbf{9}]$. Note that $\left[a^{n}, b^{m}\right]^{-1}=\left[b^{m}, a^{n}\right]$, so that every element in $\mathbf{F}^{(1)}$ has a unique expression as a product of commutators $\left\{\left[a^{n}, b^{m}\right] \mid n, m \in \mathbb{Z}, n m \neq\right.$ $0\} \cup\left\{\left[b^{n}, a^{m}\right] \mid n, m \in \mathbb{Z}, n m \neq 0\right\}$, such that $\left[a^{n}, b^{m}\right]$ and $\left[b^{m}, a^{n}\right]$ do not appear as consecutive letters. 
Let $G$ be a group. For a sequence $g_{1}, \ldots, g_{n} \in G$ we write $\prod_{i=1}^{n} g_{i}$ to denote the ordered product $g_{1} g_{2} \cdots g_{n} \in G$. Note that this is non-standard since we do not assume that the $g_{i}$ 's commute.

2.2. Some Lie theory. We denote by $S U(n)$ the group of $n \times n$ special unitary matrices and by $1_{n} \in S U(n)$ the identity matrix. The subgroup of diagonal matrices in $S U(n)$ is denoted by

$$
T:=\left\{\operatorname{diag}\left(e^{i \theta_{1}}, \ldots, e^{i \theta_{n}}\right) \mid \theta_{i} \in \mathbb{R}, \sum_{i} \theta_{i}=0\right\} .
$$

Any element in $S U(n)$ is conjugate to some element in $T$ and $T$ is called maximal torus in $S U(n)$. The Lie subalgebra of the Lie algebra $\mathfrak{s u}(n)$ corresponding to $T$ is the Cartan subalgebra

$$
\mathfrak{h}:=\left\{\operatorname{diag}\left(i \theta_{1}, \ldots, i \theta_{n}\right) \mid \theta_{i} \in \mathbb{R}, \sum_{i} \theta_{i}=0\right\} .
$$

We denote by exp: $\mathfrak{s u}(n) \rightarrow S U(n)$ the exponential map and note that its restriction to $\mathfrak{h}$ is a homomorphism exp: $\mathfrak{h} \rightarrow T$. We denote by $N(T)$ the normalizer of $T$ in $S U(n)$. The Weyl group of $S U(n)$ is

$$
W(T, S U(n))=N(T) / T \simeq S_{n}
$$

and it acts on $\mathfrak{h}$ by permutation of the coordinates. The linearization of this action yields a homomorphism $\operatorname{Ad}: \mathbb{R}\left[S_{n}\right] \rightarrow \operatorname{End}_{\mathbb{R}}(\mathfrak{h})$, which will play an important role in our study.

A basic property of word maps $\omega: S U(n) \times S U(n) \rightarrow S U(n)$ is the identity

$$
\omega\left(z u z^{*}, z v z^{*}\right)=z w(u, v) z^{*} .
$$

Hence, in order to show surjectivity, it is enough to show that $T \subset S U(n)$ lies in the image of $\omega$. We will frequently make use of this fact. For more details about compact Lie groups, see [8].

2.3. Review of known results. Let us now start with an easy observation. As mentioned, it is easy to see that the word maps are surjective for $\omega \notin \mathbf{F}^{(1)}$.

Lemma 2.1. Let $\mathbf{F}$ be the free group on two generators, $\omega \in \mathbf{F} \backslash \mathbf{F}^{(1)}$, and $n \in \mathbb{N}$. Then, the word map $\omega: S U(n) \times S U(n) \rightarrow S U(n)$ is surjective.

Proof. We write

$$
\omega(a, b)=a^{n_{1}} b^{m_{1}} a^{n_{2}} b^{m_{2}} \ldots a^{n_{k}} b^{m_{k}}
$$

with $n_{i}, m_{i} \in \mathbb{Z}$ and note that $\sum_{i} n_{i} \neq 0$ or $\sum_{i} m_{i} \neq 0$. Without loss of generality $\sum_{i} n_{i}=k \neq 0$. If $g \in T$, then $g=h^{k}$ for some $h \in T$. Indeed, since the exponential map exp: $\mathfrak{h} \rightarrow T$ is a surjective homomorphism, we can take $\bar{g} \in \mathfrak{h}$ to be some preimage of $g$ and set $h:=\exp (\bar{g} / k)$. Then $\omega\left(h, 1_{n}\right)=h^{k}=g$. We conclude that $\omega$ is a surjective map.

We will now explain the Gotô's proof of the main result from $[\mathbf{3}, \mathbf{1 1}]$ - in the case of $G=S U(n)$.

Theorem 2.2 (Gotô, Tôyama). Let $n \in \mathbb{N}$. The word map $\omega: S U(n) \times S U(n) \rightarrow S U(n)$ with $\omega(a, b)=[a, b]$ is surjective. 
ABDELRHMAN ELKASAPY AND ANDREAS THOM

Proof. For the permutation $\sigma=(1,2, \ldots, n) \in S_{n}$, it is easy to see that $\operatorname{Ad}(\sigma-1)$ is a vector space automorphism of $\mathfrak{h}$. Indeed, it is well-known that the eigenvalues of $\operatorname{Ad}(\sigma) \operatorname{acting}$ on $\mathfrak{h}$ are $\{\exp (2 \pi i l / n) \mid 1 \leq l \leq n-1\}$.

Now, let $g \in T$ be arbitrary. Since the exponential map exp $: \mathfrak{h} \rightarrow T$ is surjective, there exists $\bar{g} \in \mathfrak{h}$ such that $g=\exp (\bar{g})$. Since $\operatorname{Ad}(\sigma-1)$ is automorphism of $\mathfrak{h}$, there is $\bar{h} \in \mathfrak{h}$ such that $\bar{g}=\operatorname{Ad}(\sigma-1)(h)$. Then, setting $h:=\exp (\bar{h})$ we get:

$$
g=\exp \bar{g}=\exp (\operatorname{Ad}(\sigma-1)(h))=\exp (\operatorname{Ad}(\sigma) h) \exp (-h)=\sigma h \sigma^{-1} h^{-1}=[\sigma, h] .
$$

Now, the permutation matrix $\sigma$ might not be in $S U(n)$, however if $\operatorname{det}(\sigma)=-1$, then we just replace $\sigma$ by $\exp (\pi i / n) \sigma \in S U(n)$. This proves the claim.

REMARK 2.3. Note that Gotô's proof shows the stronger statement that there exists a conjugacy class $C \subset S U(n)$ such that $C^{2}=S U(n)$. Indeed, for odd $n \in \mathbb{N}$ just take $C$ to be the conjugacy class of $\sigma$ and note that $\sigma^{-1} \in C$; similarly for $\exp (\pi i / n) \sigma$ if $n$ is even. In the world of non-abelian finite simple groups, this is known as Thompson's conjecture.

The idea in Gotô's proof depends on finding a suitable Laurent polynomial $p(t) \in \mathbb{Z}\left[t, t^{-1}\right]$ and a suitable element $\sigma \in W(T)$ in the Weyl group of the maximal torus such that $\operatorname{Ad}(p(\sigma))$ is a vector space automorphism of the Cartan subalgebra. In the case of the commutator word $\omega=[a, b]$, we take $\sigma=(1,2, \ldots, n) \in S_{n}$ and $p(t)=t-1$. Our goal is to extend the method to cover more elements in $\mathbf{F}$.

\section{The main result}

In this section, we want to associate to $\omega \in \mathbf{F}^{(1)}$ a polynomial $p_{\omega}$ which can be used in an argument analogous to the one in Gotô's proof. We define a homomorphism $p_{\omega}: \mathbf{F}^{(1)} \rightarrow \mathbb{Z}\left[t, t^{-1}\right]$ by setting

$$
p_{\left[a^{n}, b^{m}\right]}(t)=m\left(t^{n}-1\right), \quad \forall n, m \in \mathbb{Z}, n m \neq 0 .
$$

Note that this is well-defined since $\left\{\left[a^{n}, b^{m}\right] \mid n, m \in \mathbb{Z}, n m \neq 0\right\}$ generates $\mathbf{F}^{(1)}$ freely, see Section 2.1. Since $\mathbb{Z}\left[t, t^{-1}\right]$ abelian, $p_{\omega}=0$ for all $\omega \in \mathbf{F}^{(2)}$.

LEMma 3.1. Let $\mathbf{F}$ be the free group on two generators and let $\omega \in \mathbf{F}^{(1)}$. If $p_{\omega}(\exp (2 \pi l i / n)) \neq 0$ for $1 \leq l \leq n-1$, then the word map $\omega: S U(n) \times S U(n) \rightarrow S U(n)$ is surjective.

Proof. We write

$$
\omega=\left[a^{n_{1}}, b^{m_{1}}\right]^{\varepsilon_{1}} \cdots\left[a^{n_{k}}, b^{m_{k}}\right]^{\varepsilon_{k}}
$$

with $n_{i}, m_{i} \in \mathbb{Z}$ and $\varepsilon_{i} \in\{ \pm 1\}$. Then,

$$
p_{\omega}(t)=\sum_{i=1}^{k} \varepsilon_{i} m_{i}\left(t^{n_{i}}-1\right) .
$$

Let $g \in T$ be arbitrary and let $\bar{g} \in \mathfrak{h}$ be such that $\exp (\bar{g})=g$. Let $\sigma=(1,2, \cdots, n) \in W(T)$. By assumption $\operatorname{Ad}\left(p_{\omega}(\sigma)\right)$ is invertible in $\operatorname{End}_{\mathbb{R}}(\mathfrak{h})$. Let $\bar{h} \in \mathfrak{h}$ be such that $\left.\operatorname{Ad}\left(p_{\omega}(\sigma)\right)(\bar{h})\right)=\bar{g}$ and set 
$h:=\exp (\bar{h})$. We claim that $\omega(\sigma, h)=g$. Indeed,

$$
\begin{aligned}
\omega(\sigma, h) & =\left[\sigma^{n_{1}}, h^{m_{1}}\right]^{\varepsilon_{1}} \cdots\left[\sigma^{n_{k}}, h^{m_{k}}\right]^{\varepsilon_{k}} \\
& =\prod_{i=1}^{k} \exp \left(\operatorname{Ad}\left(\sigma^{n_{i}}\right)\left(\varepsilon_{i} m_{i} \bar{h}\right)\right) \exp \left(-\varepsilon_{i} m_{i} \bar{h}\right) \\
& =\exp \left(\operatorname{Ad}\left(p_{\omega}(\sigma)(\bar{h})\right)\right) \\
& =g
\end{aligned}
$$

If $n$ is even, then we must replace $\sigma$ by $\exp (\pi i / n) \sigma \in S U(n)$. This proves the claim.

Corollary 3.2. Let $\mathbf{F}$ be the free group on two generators and let $\omega \in \mathbf{F}^{(1)}$. If $p_{\omega} \neq 0$, then there exists an integer $k \in \mathbb{N}$, such that for all $n \in \mathbb{N}$ with $\operatorname{lpf}(n) \geq k$, the word map $\omega: S U(n) \times S U(n) \rightarrow$ $S U(n)$ is surjective.

Proof. Assume that $p_{\omega}(t)=\sum_{i \in \mathbb{Z}} a_{i} t^{i} \neq 0$. Let $S:=\left\{i \in \mathbb{Z} \mid a_{i} \neq 0\right\}$ and set $k:=$ $\max S-\min S$. Let $n \in \mathbb{N}$ and assume that $\xi:=\exp (2 \pi i l / n)$ for some $1 \leq l \leq n-1$ satisfies $p_{\omega}(\xi)=0$. Let $d$ be the degree of the minimal polynomial $m_{\xi}$ of $\xi$. If $\xi$ is a primitive $m$-th root of unity, then $m \mid n$ and $d=\varphi(m)$, where $\varphi$ denotes Euler's $\varphi$-function. For some prime $p$ which divides $n$, we must have $(p-1) \mid \varphi(m)$ and hence $\operatorname{lpf}(n)-1 \leq d$. Since $p_{\omega}(t)$ has rational coefficients, we also get that $m_{\xi} \mid p_{\omega}$ in the ring $\mathbb{Q}\left[t, t^{-1}\right]$ and hence $d \leq k$. Hence, if the assumption of Lemma 3.1 fails then $\operatorname{lpf}(n)-1 \leq k$. This proves the claim.

Let us discuss some examples to see how the previous results can be applied and what their limitations are.

EXAMPLE 3.3. The word map $\omega: S U(n) \times S U(n) \rightarrow S U(n) \omega(a, b)=[a, b]^{2}$ is surjective for all $n \in \mathbb{N}$. Indeed, $p_{\omega}(t)=2(t-1)$ and $\operatorname{Ad}\left(p_{\omega}(\sigma)\right)$ is a vector space automorphism of $\mathfrak{h}$ for $\sigma=(1,2, \ldots, n)$.

EXAmple 3.4. The word map $\omega: S U(n) \times S U(n) \rightarrow S U(n)$ for $\omega(a, b)=a^{2} b a^{-1} b a^{-1} b^{-2}$ is surjective for all $n \in \mathbb{N}$. We have $p_{\omega}(t)=t^{2}+t-2$ and $\operatorname{Ad}\left(p_{\omega}(\sigma)\right)$ is a vector space automorphism of $\mathfrak{h}$ for $\sigma=(1,2, \ldots, n)$.

It is easy to see that $p_{\omega}$ vanishes for $\omega(a, b)=[a, b]\left[a, b^{-1}\right]$ even though $\omega \notin \mathbf{F}^{(2)}$. In this case we can still apply the method since we may interchange the role of $a$ and $b$ and note that $p_{\omega^{\prime}} \neq 0$ for $\omega(a, b)=[b, a]\left[b, a^{-1}\right]$. However, for $\omega(a, b)=[a, b]\left[a, b^{-1}\right]\left[a^{-1}, b\right]\left[a^{-1}, b^{-1}\right]$ no such trick helps and we have to consider more complicated Nielsen transformations and their effect on our polynomial. We will show that for each $\omega \notin \mathbf{F}^{(2)}$, there exists a basis for $\mathbf{F}$, such that with respect to the new basis, $p_{\omega} \neq 0$. Any base change is induced by a sequence of Nielsen transformations. In Proposition 3.6 we study in detail how the base change $a \mapsto a b, b \mapsto b$ can be expressed in the natural basis of $\mathbf{F}^{(1)}$.

For $x, y \in G$, we use the notation ${ }^{y} x:=y x y^{-1}$. Note that this convention implies ${ }^{z}\left({ }^{y} x\right)={ }^{z y} x$ and ${ }^{z}(x y)={ }^{z} x^{z} y$ as expected. It is well-known that for $x, y, z \in G$ we get:

$$
[x, y z]=[x, y] \cdot{ }^{y}[x, z] \text { and }[x y, z]={ }^{x}[y, z] \cdot[x, z] .
$$


From now on let us write $c:=a b$. Note that the set $\{c, b\}$ is a basis for $\mathbf{F}$. Our next goal is to express $\left[a^{n}, b^{m}\right]$ in terms of the commutators $\left[c^{n}, b^{m}\right]$, i.e. we want to determine the effect of the base change on the natural basis for $\mathbf{F}^{(1)}$. We will need the following lemma.

Lemma 3.5. Let $G$ be a group, $a, b \in G$ and $c:=a b$. Let $n, m \in \mathbb{Z}$. Then, the following identities hold:

$$
{ }^{a}\left[c^{n}, b^{m}\right]=\left[c, b^{-1}\right]\left[b^{-1}, c^{n+1}\right]\left[c^{n+1}, b^{m-1}\right]\left[b^{m-1}, c\right] .
$$

and

$$
a^{-1}\left[c^{n}, b^{m}\right]=\left[b, c^{n-1}\right]\left[c^{n-1}, b^{m+1}\right]\left[b^{m+1}, c^{-1}\right]\left[c^{-1}, b\right]
$$

PROOF. In order to prove (2), we compute

$$
\begin{aligned}
{ }^{a}\left[c^{n}, b^{m}\right] & =c b^{-1}\left[c^{n}, b^{m}\right] b c^{-1} \\
& =c b^{-1} c^{n} b^{m} c^{-n} b^{-m} b c^{-1} \\
& =c b^{-1} c^{-1} b b^{-1} c^{n+1} b c^{-(n+1)} c^{n+1} b^{m-1} c^{-(n+1)} b^{-(m-1)} b^{m-1} c b^{-(m-1)} c^{-1} \\
& =\left[c, b^{-1}\right]\left[b^{-1}, c^{n+1}\right]\left[c^{n+1}, b^{m-1}\right]\left[b^{m-1}, c\right] .
\end{aligned}
$$

For (3) we compute

$$
\begin{aligned}
a^{-1}\left[c^{n}, b^{m}\right] & =b c^{-1} c^{n} b^{m} c^{-n} b^{-m} c b^{-1} \\
& =b c^{n-1} b^{-1} c^{-(n-1)} \cdot c^{n-1} b^{m+1} c^{-(n-1)} b^{-(m+1)} \cdot b^{m+1} c^{-1} b^{-(m+1)} c \cdot c^{-1} b c b^{-1} \\
& =\left[b, c^{n-1}\right]\left[c^{n-1}, b^{m+1}\right]\left[b^{m+1}, c^{-1}\right]\left[c^{-1}, b\right]
\end{aligned}
$$

This finishes the proof.

Taking the inverse of Equation (2) we obtain for all $n, m \in \mathbb{Z}$ :

$$
{ }^{a}\left[b^{m}, c^{n}\right]=\left[c, b^{m-1}\right]\left[b^{m-1}, c^{n+1}\right]\left[c^{n+1}, b^{-1}\right]\left[b^{-1}, c\right] .
$$

and

$$
a^{-1}\left[b^{m}, c^{n}\right]=\left[b, c^{-1}\right]\left[c^{-1}, b^{m+1}\right]\left[b^{m+1}, c^{n-1}\right]\left[c^{n-1}, b\right] .
$$

We are now ready to state and prove the technical heart of our computations.

Proposition 3.6. Let $G$ be a group, $a, b \in G, c:=a b$ and let $m \in \mathbb{Z}$. If Then the following equations holds:

$$
\begin{gathered}
{\left[a^{n}, b^{m}\right]=\prod_{i=1}^{n-1}\left[c^{i}, b^{-i}\right]\left[b^{-i}, c^{i+1}\right] \cdot \prod_{i=1}^{n}\left[c^{n+1-i}, b^{m-n+i}\right]\left[b^{m-n+i}, c^{n-i}\right], \quad n \geq 1 .} \\
{\left[a^{-n}, b^{m}\right]=\prod_{i=1}^{n}\left[c^{1-i}, b^{i}\right]\left[b^{i}, c^{-i}\right] \cdot \prod_{i=1}^{n}\left[c^{-(n+1)+i}, b^{n+m+1-i}\right]\left[b^{n+m+1-i}, c^{-n+i}\right], \quad n \geq 1 .}
\end{gathered}
$$

The main feature of the formulas above is the following. For $n \geq 1$, the powers of $b$ that appear expressing $\left[a^{n}, b^{m}\right]$ in the new basis will all be less or equal $\max \{-1, m\}$. At the same time, the 
powers of $a$ range between 1 and $n$. The powers of $b$ that appear when expressing $\left[a^{-n}, b^{m}\right]$ will be less or equal $\max \{n, n+m\}$ and if $m \geq 1$, then $\left[c^{-n}, b^{n+m}\right]\left[b^{n+m}, c^{-n+1}\right]$ will appear exactly once. We will use this consequence in the proof of our main result.

Proof of Proposition 3.6: We prove the claim (6) by induction on $n \in \mathbb{N}$. The claim is obviously true for $n=1$, since $\left[a, b^{m}\right]=\left[c, b^{m}\right]$. Let $m \in \mathbb{N}$ and assume that the claim (6) is known for the pair $(n-1, m)$. We compute

$$
\begin{aligned}
& {\left[a^{n}, b^{m}\right] } \\
= & {\left[a a^{n-1}, b^{m}\right] } \\
\stackrel{(1)}{=} & { }^{a}\left[a^{n-1}, b^{m}\right]\left[a, b^{m}\right] \\
\stackrel{(6)}{=} & \left(\prod_{i=1}^{n-2}{ }^{a}\left[c^{i}, b^{-i}\right]^{a}\left[b^{-i}, c^{i+1}\right] \cdot \prod_{i=1}^{n-1}\left[c^{n+1-i}, b^{m-n+i}\right]^{a}\left[b^{m-n+i}, c^{n-i}\right]\right)\left[c, b^{m}\right] \\
\stackrel{(2)+(4)}{=} & \prod_{i=1}^{n-2}\left[c, b^{-1}\right]\left[b^{-1}, c^{i+1}\right]\left[c^{i+1}, b^{-i-1}\right]\left[b^{-i-1}, c^{i+2}\right]\left[c^{i+2}, b^{-1}\right]\left[b^{-1}, c\right] \cdot \\
& \prod_{i=1}^{n-1}\left[c, b^{-1}\right]\left[b^{-1}, c^{n-i+2}\right]\left[c^{n-i+2}, b^{m-n+i-1}\right]\left[b^{m-n+i-1}, c^{n-i+1}\right]\left[c^{n-i+1}, b^{-1}\right]\left[b^{-1}, c\right] . \\
& {\left[c, b^{m}\right] } \\
& \prod_{i=1}^{n-1}\left[c^{i}, b^{-i}\right]\left[b^{-i}, c^{i+1}\right] \cdot \prod_{i=1}^{n}\left[c^{n+1-i}, b^{m-n+i}\right]\left[b^{m-n+i}, c^{n-i}\right] .
\end{aligned}
$$

Now, we prove the claim (7) by induction on $n$. Again, the claim is true for $n=1$ since

$$
\left[a^{-1}, b^{m}\right]=a^{-1} b^{m} a b^{-m}=b c^{-1} b^{m} c b^{-1} b^{-m}=b c^{-1} b^{-1} c c^{-1} b^{m+1} c b^{-(m+1)}=\left[b, c^{-1}\right]\left[c^{-1}, b^{m+1}\right] .
$$

Let us assume that the claim (7) is known for the pair $(n-1, m)$. We compute:

$$
\begin{aligned}
& {\left[a^{-n}, b^{m}\right] } \\
= & {\left[a^{-1} a^{-(n-1)}, b^{m}\right] } \\
\stackrel{(1)}{=} & a^{-1}\left[a^{-(n-1)}, b^{m}\right] \cdot\left[a^{-1}, b^{m}\right] \\
\stackrel{(7)}{=} & \prod_{i=1}^{n-1} a^{-1}\left[c^{1-i}, b^{i}\right]^{a^{-1}}\left[b^{i}, c^{-i}\right] \cdot \prod_{i=1}^{n-1} a^{-1}\left[c^{-(n+1)+i}, b^{n+m+1-i}\right]^{a^{-1}}\left[b^{n+m+1-i}, c^{-n+i}\right] . \\
& {\left[b, c^{-1}\right]\left[c^{-1}, b^{m+1}\right] } \\
\stackrel{(3)+(5)}{=} & \prod_{i=1}^{n-1}\left[b, c^{-i}\right]\left[c^{-i}, b^{i+1}\right]\left[b^{i+1}, c^{-1}\right]\left[c^{-1}, b\right]\left[b, c^{-1}\right]\left[c^{-1}, b^{i+1}\right]\left[b^{i+1}, c^{-i-1}\right]\left[c^{-i-1}, b\right] . \\
& \prod_{i=1}^{n-1}\left[b, c^{-n+i-2}\right]\left[c^{-n+i-2}, b^{n+m-i+2}\right]\left[b^{n+m-i+2}, c^{-n+i-1}\right]\left[c^{-n+i-1}, b\right] . \\
& {\left[b, c^{-1}\right]\left[c^{-1}, b^{m+1}\right] }
\end{aligned}
$$




$$
\begin{aligned}
= & \prod_{i=1}^{n-1}\left[b, c^{-i}\right]\left[c^{-i}, b^{i+1}\right]\left[b^{i+1}, c^{-i-1}\right]\left[c^{-i-1}, b\right] \cdot \\
& \prod_{i=1}^{n-1}\left[b, c^{-n+i-2}\right]\left[c^{-n+i-2}, b^{n+m-i+2}\right]\left[b^{n+m-i+2}, c^{-n+i-1}\right]\left[c^{-n+i-1}, b\right] . \\
& {\left[b, c^{-1}\right]\left[c^{-1}, b^{m+1}\right] } \\
= & \prod_{i=1}^{n}\left[c^{1-i}, b^{i}\right]\left[b^{i}, c^{-i}\right] \cdot \prod_{i=1}^{n}\left[c^{-(n+1)+i}, b^{n+m+1-i}\right]\left[b^{n+m+1-i}, c^{-n+i}\right] .
\end{aligned}
$$

This proves the claim.

The following proposition shows that our previous computations are enough to deal with some more complicated words.

Proposition 3.7. Let $n \in \mathbb{N}$. The word map

$$
\omega: S U(n) \times S U(n) \rightarrow S U(n)
$$

for $\omega(a, b)=[a, b]\left[a, b^{-1}\right]\left[a^{-1}, b\right]\left[a^{-1}, b^{-1}\right]$ is surjective.

Proof. Using Proposition 3.6 we can write $\omega$ in the following form:

$$
\omega=[c, b]\left[c, b^{-1}\right]\left[b, c^{-1}\right]\left[c^{-1}, b^{2}\right]\left[b, c^{-1}\right]
$$

where $c=a b$. Indeed, $[a, b]=[c, b],\left[a, b^{-1}\right]=\left[c, b^{-1}\right],\left[a^{-1}, b^{ \pm 1}\right]=\left[b, c^{-1}\right]\left[c^{-1}, b^{1 \pm 1}\right]$ and hence $\omega=[c, b]\left[c, b^{-1}\right]\left[b, c^{-1}\right]\left[c^{-1}, b^{2}\right]\left[b, c^{-1}\right]$ as claimed. Now, we may compute $p_{\omega}$ with respect to the basis $\{b, c\}$ and obtain

$$
p_{\omega}(t)=-(t-1)-\left(t^{-1}-1\right)-(t-1)+\left(t^{2}-1\right)-(t-1)=t^{2}-3 t-t^{-1}+3 \neq 0 .
$$

It is easy to see that if a root of unity $\xi$ satisfies $p_{\omega}(\xi)=0$, then $\xi=1$. Hence, Lemma 3.1 implies that the word map associated with $\omega$ is surjective for all $n \in \mathbb{N}$.

The key observation is that in the expression for $\left[a^{i}, b^{j}\right]$ in terms of $\left\{\left[c^{n}, b^{m}\right] \mid n, m \in \mathbb{Z}, n m \neq 0\right\}$ can be used to isolate certain exponents. This will be used to show that for $\omega \notin \mathbf{F}^{(2)}$, there is always some basis such that $p_{\omega} \neq 0$.

Proposition 3.8. Let $\mathbf{F}$ be the free group on two generators $\{a, b\}$ and $\omega \in \mathbf{F}^{(1)}$. If $\omega \notin \mathbf{F}^{(2)}$, then there exists an basis of $\mathbf{F}$ such that $p_{\omega}(t) \neq 0$, when computed with respect to this basis.

Proof. The idea is to use the mechanism that is hidden in the proof of Proposition 3.7. Let $\omega \in \mathbf{F}^{(1)}$ and $\omega \notin \mathbf{F}^{(2)}$. Let us write

$$
\omega=\left[a^{n_{1}}, b^{m_{1}}\right]^{\nu_{1}}\left[a^{n_{2}}, b^{m_{2}}\right]^{\nu_{2}} \cdots\left[a^{n_{k}}, b^{m_{k}}\right]^{\nu_{k}}
$$

Since $\omega \mapsto p_{\omega} \in \mathbb{Z}\left[t, t^{-1}\right]$ is a homomorphism for any basis of $\mathbf{F}$, we may freely rearrange the commutators in the product above. Moreover, we may assume that $\left(n_{i}, m_{i}\right) \neq\left(n_{j}, m_{j}\right)$ for $i \neq j$ and $\nu_{i} \in \mathbb{Z} \backslash\{0\}$. 
Let $n:=\max \left\{\left|n_{1}\right|, \ldots,\left|n_{k}\right|\right\}$. Without loss of generality we can assume that $n=-n_{1}$. Indeed, exchanging $a$ with $a^{-1}$, exchanges $n_{i}$ with $-n_{i}$, so that we may assume that $n_{i}<0$. Reordering the product allows to assume that $n=-n_{1}$. Again, reordering does not change $p_{\omega}$ in any basis, since only the class of $\omega$ in $\mathbf{F}^{(1)} / \mathbf{F}^{(2)}$ matters in our computation. In addition, we may assume that there exists $k^{\prime} \in \mathbb{N}$ such that $n=n_{1}=n_{2}=\cdots=n_{k^{\prime}}$ and $n \neq n_{l}$ for $l>k^{\prime}$. Without loss generality, we have $m_{1}>m_{2}>\cdots>m_{k^{\prime}}$ and set $m:=m_{1}$. Upon possibly replacing $b$ by $b^{-1}$, we may assume that $m>0$.

Let us now set $c:=a b \in \mathbf{F}$. We will now analyze how $\omega$ is written in terms of the basis $\{c, b\}$. By Proposition 3.6, each factor $\left[a^{-n}, b^{m_{i}}\right]$ of $\omega$ contains factors $\left[c^{-n}, b^{n}\right]^{-1}$ and $\left[c^{-n}, b^{n+m_{i}}\right]$ and these are the only factors in $\omega$ of the form $\left[c^{-n^{\prime}}, b^{k}\right]$ for some $k \in \mathbb{Z}$ and $n^{\prime} \geq n$. Repeating this process, we can set $c_{q}:=a b^{q}$ and consider the basis $\left\{c_{q}, b\right\}$. With respect to this basis $\omega$ will contain a factor of the form $\left[c_{q}^{-n}, b^{q n+m}\right]$. From Proposition 3.6 and the remarks after its statement, we conclude

that for $q \in \mathbb{N}$ high enough, the factor $\left[c_{q}^{-n}, b^{q n+m}\right]\left[b^{q n+m}, c_{q}^{-n+1}\right]$ will be the only appearance of $b^{q n+m}$. Hence, computing $p_{\omega}$ with respect to the basis $\left\{b, c_{q}\right\}$, the coefficient of $t^{q n+m}$ will be non-zero. This proves the claim.

We are now ready to prove Theorem 1.1.

Proof of Theorem 1.1: Let $\omega \in \mathbf{F} \backslash \mathbf{F}^{(2)}$ be arbitrary. If $\omega \notin \mathbf{F}^{(1)}$, then Lemma 2.1 proves the claim. Hence, we may assume $\omega \in \mathbf{F}^{(1)}$ and $\omega \notin \mathbf{F}^{(2)}$. By Proposition 3.8, there exists a basis of $\mathbf{F}$ such that $p_{\omega}(t) \neq 0$. The claim follows from Corollary 3.2.

\section{Special families of words and open problems}

4.1. Engel words. In this last section we study Engel words and show that the associated word maps are always surjective. Corresponding results for finite simple groups were proved in $[\mathbf{1}]$.

Definition 4.1. Let $\mathbf{F}$ be the free group on two generators $\{a, b\}$. The $k$-th Engel word $e_{k}(a, b) \in \mathbf{F}$ is defined recursively by the equations:

$$
\begin{aligned}
& e_{0}(a, b)=a, \\
& e_{k}(a, b)=\left[e_{k-1}, b\right], \quad k \geq 1 .
\end{aligned}
$$

For a group $G$, the corresponding map $e_{k}: G \times G \rightarrow G$ is called the $k$-th Engel word map. We are now ready to prove Theorem 1.2

Proof of Theorem 1.2: We want to compute $p_{e_{k}}$ with respect to the basis $\{b, a\}$. First of all, it is easy to see that ${ }^{b}\left[b^{m}, a^{n}\right]=\left[b^{m+1}, a^{n}\right]\left[b, a^{n}\right]^{-1}$ for $n, m \in \mathbb{Z}$. Indeed, we just compute

$$
\begin{aligned}
{ }^{b}\left[b^{m}, a^{n}\right] & =b b^{m} a^{n} b^{-m} a^{-n} b^{-1} \\
& =b^{m+1} a^{n} b^{-(m+1)} a^{-n} a^{n} b a^{-n} b^{-1} \\
& =\left[b^{m+1}, a^{n}\right]\left[b, a^{n}\right]^{-1} .
\end{aligned}
$$


This shows that if $p_{\omega}(t)=\sum_{i} a_{i} t^{i}$, then $p_{b} \omega(t)=\sum_{i} a_{i} t\left(t^{i}-1\right)=t p_{\omega}(t)-t p_{\omega}(1)$. Hence,

$$
p_{[\omega, b]}(t)=p_{\omega}(t)-p_{b}(t)=(1-t) p_{\omega}(t)+t p_{\omega}(1) .
$$

Since $p_{e_{1}}(t)=p_{[b, a]^{-1}}(t)=1-t$ we conclude that $p_{e_{k}}(t)=(1-t)^{k}$ for all $k \in \mathbb{N}$. Lemma 3.1 implies the claim.

4.2. Open problems. It is clear that the method presented in this paper has serious limitations and cannot possible work for words $\omega \in \mathbf{F}^{(2)}$. A first non-trivial case is $\omega(a, b)=$ $\left[[a, b],\left[a^{2}, b^{2}\right]\right]$. It is unknown to us if Larsen's question has a positive answer for this word.

Question 4.2. Let $\mathbf{F}$ be the free group on two generators $\{a, b\}$ and let $\omega=\left[[a, b],\left[a^{2}, b^{2}\right]\right]$. Is the associated word map $\omega: S U(n) \times S U(n) \rightarrow S U(n)$ surjective for all but finitely many $n \in \mathbb{N}$.

If $\omega \notin \mathbf{F}^{(2)}$ it would be desirable to find out if the restrictions on $n \in \mathbb{N}$ in Theorem 1.1 are necessary. We are not aware of a word $\omega \notin \mathbf{F}^{(2)}$, where the associated word map is not surjective for all $n \in \mathbb{N}$. In order to understand this problem, we need to understand the map $\omega \mapsto p_{\omega}$ more directly. We can endow $\mathbb{Z}\left[t, t^{-1}\right]$ with a $\mathbb{Z} \mathbf{F}$-module structure such that $a \cdot f(t)=t f(t)$ and $b \cdot f(t)=f(t)$ for all $f(t) \in \mathbb{Z}\left[t, t^{-1}\right]$. The quotient $\mathbf{F}^{(1)} / \mathbf{F}^{(2)}$ is also a $\mathbb{Z} \mathbf{F}$-module, where the module structure is induced from the conjugation action. Since $p_{\omega}$ is well-defined on $\mathbf{F}^{(1)} / \mathbf{F}^{(2)}$, it is natural to study the induced map

$$
\bar{p}: \mathbf{F}^{(1)} / \mathbf{F}^{(2)} \rightarrow \mathbb{Z}\left[t, t^{-1}\right] .
$$

Lemma 4.3. The map $\bar{p}$ is a homomorphism of $\mathbb{Z} \mathbf{F}$-modules.

Proof. We denote the class of $\left[a^{n}, b^{m}\right]$ in $\mathbf{F}^{(1)} / \mathbf{F}^{(2)}$ by $\xi_{n, m}$. Hence, $\bar{p}\left(\xi_{n, m}\right)=m\left(t^{n}-\right.$ 1) by definition. It follows from the equations ${ }^{a}\left[a^{n}, b^{m}\right]=\left[a^{n+1}, b^{m}\right]\left[a, b^{m}\right]^{-1}$ and ${ }^{b}\left[a^{n}, b^{m}\right]=$ $\left[a^{n}, b\right]^{-1}\left[a^{n}, b^{m+1}\right]$ that $\bar{p}\left(a \cdot \xi_{n, m}\right)=\bar{p}\left(\xi_{n+1, m}-\xi_{1, m}\right)=m\left(t^{n+1}-t\right)=t \cdot \bar{p}\left(\xi_{n, m}\right)$ and $\bar{p}\left(b \cdot \xi_{n, m}\right)=$ $\bar{p}\left(\xi_{n, m+1}-\xi_{n, 1}\right)=m\left(t^{n}-1\right)=\bar{p}\left(\xi_{n, m}\right)$. This finishes the proof.

For $\xi \in \mathbf{F}^{(1)} / \mathbf{F}^{(2)}$ and a general automorphism $\alpha \in \operatorname{Aut}(\mathbf{F})$, the relation between $\bar{p}(\xi)$ and $\bar{p}(\alpha(\xi))$ remains obscure.

QUestion 4.4. Let $\xi \in \mathbf{F}^{(1)} / \mathbf{F}^{(2)}$. Is there an automorphism $\alpha \in \operatorname{Aut}(\mathbf{F})$ such that the only root of the polynomial $\bar{p}(\alpha(\xi))$ which is a root of unity is equal to one.

A positive answer to this question would remove the restrictions on $n \in \mathbb{N}$ in Theorem 1.1.

\section{Acknowledgment}

The research of A.T. was supported by ERC. A.E. wants to thank the IMPRS Leipzig and the MPI-MIS Leipzig for support and an excellent research environment.

\section{References}

[1] Tatiana Bandman, Fritz Grunewald, and Shelly Garion, On the Surjectivity of Engel Words on PSL $(2, q)$, arxiv:1008.1397, to appear in Journal of Groups, Geometry, and Dynamics. $\uparrow 2,9$ 
[2] Amand Borel, On free subgroups of semisimple groups, Enseign. Math. (2) 29 (1983), no. 1-2, 151-164. $\uparrow 1$

[3] Morikuni Gotô, A theorem on compact semi-simple groups, J. Math. Soc. Japan 1 (1949), 270-272. $\uparrow 2,3$

[4] Michael Larsen, Word maps have large image, Israel J. Math. 139 (2004), 149-156. $\uparrow 1$

[5] Michael Larsen and Aner Shalev, Word maps and Waring type problems, J. Amer. Math. Soc. 22 (2009), no. 2, 437-466. $\uparrow 1$

[6] Michael Larsen, Aner Shalev, and Pham Huu Tiep, The Waring problem for finite simple groups, Ann. of Math. (2) 174 (2011), no. 3, 1885-1950. $\uparrow 1$

[7] Martin W. Liebeck, E. A. O'Brien, Aner Shalev, and Pham Huu Tiep, The Ore conjecture, J. Eur. Math. Soc. 12 (2010), no. 4, 939-1008. $\uparrow 1$

[8] Mark R. Sepanski, Compact Lie groups, Graduate Texts in Mathematics, vol. 235, Springer, New York, 2007. $\uparrow 3$

[9] Jean-Pierre Serre, Trees, Springer Monographs in Mathematics, Springer-Verlag, Berlin, 2003. $\uparrow 2$

[10] Andreas Thom, Convergent sequences in discrete groups, arXiv:1003.4093, to appear in Can. Math. Bulletin. $\uparrow 1$

[11] Hiraku Tôyama, On commutators of matrices, Kōdai Math. Sem. Rep., 1 (1949), no. 5-6, 1-2. $\uparrow 2,3$

Abdelrhman Elkasapy, MPI-Mis, Inselstrasse 22, 04103 Leipzig, Germany, and Mathematics Department, South Valley University, Qena, Egypt

E-mail address: elkasapy@mis.mpg.de

Andreas Thom, Univ. Leipzig, PF 100920, 04009 LeipZig, Germany

E-mail address: andreas.thom@math.uni-leipzig.de 